\title{
Is nephrostomy tube a rule following all Percutaneous Nephrolithotomy (PCNL)? A prospective randomized study
}

Pawan Raj Chalise ${ }^{1}$, Bhojraj Luitel ${ }^{1}$, Suman Chapagain ${ }^{1}$, Sujeet Poudyal ${ }^{1}$, Prem Raj Gyawali ${ }^{1}$, Uttam Kumar Sharma1, Bhola Raj Joshi²

${ }^{1}$ Department of Urology and Kidney Transplant Surgery, TU Teaching Hospital, Institute of Medicine, Tribhuvan University

${ }^{2}$ Department of Urology, Nepal Medical College Teaching Hospital

Correspondence: Dr Pawan Raj Chalise, Lecturer, Department of Urology and Kidney Transplant Surgery, TU Teaching Hospital

Email: pawan_rc@yahoo.com

\section{Abstract}

Introduction: Exit strategy at the end of percutaneous nephrolithotomy (PCNL) differs from center to center and patient to patient. Standard PCNL has been practiced so far with minor postoperative morbidities. Tubeless PCNL, which obviates most of the nephrostomy related morbidities, has been challenged for its safety. So this study was conducted to compare the safety and morbidity of tubeless PCNL with standard PCNL.

Methods: Patients who had undergone PCNL, were randomized into group 1 (standard) and group 2 (tubeless) using computer generated random table. In group 1, nephrostomy tube was placed at the end of the procedure and tubes were omitted in group 2 patients. All preoperative, intraoperative and postoperative parameters were recorded and compared in between the groups.

Results: Ninety six PCNLs were randomized into group 1 (47 patients) and group 2 (49 patients). Patients' characteristics including age, sex, comorbidities, preoperative parameters, size and number of stones and mean operation time were comparable in between the groups. The incidence of postoperative fever, pain and analgesic requirement and urinary leak were found more in group 1 patients. The incidence of postoperative complications and events were comparable in both the groups except for blood transfusion. The mean length of postoperative hospital stay for patients in group 2 was significantly low as compared to group 1 .

Conclusion: Tubeless PCNL is safe and has less morbidity as compared to standard PCNL in selected cases.

Key Words: Morbidity; PCNL, Standard PCNL; Tubeless PCNL.

\section{Introduction}

Percutaneous Nephrolithotomy (PCNL) is one of the most commonly performed surgical procedures in urologic practice for management of upper urinary tract lithiasis. Exit strategy at the end of the procedure differs from center to center and patient to patients. Standard and tubeless PCNL have been practiced, but none is found superior to other.
Over the past few decades, lots of criticisms appeared in the literature for standard exit strategy (placement of nephrostomy tube) due to its invasiveness and reported morbidities. ${ }^{1-4}$ Many urologist realized that substantial postoperative pain and morbidity after PCNL are caused by nephrostomy tubes and attempts have been made to 
modify standard PCNL by avoiding use of a nephrostomy tube altogether with internal ureteral (JJ) stent for drainage of urine after surgery, termed as tubeless PCNL. Bellman et al, in 1997, challenged the requirement for the routine placement of a nephrostomy tube after PCNL and became the first to report the safety of tubeless PCNL. ${ }^{5}$

Percutaneous nephrolithotomy service was started at authors' institute since 2007 and standard PCNL were practiced. The present study was conducted to evaluate the outcome, safety and morbidity of tubeless PCNL over standard PCNL.

\section{Methods}

A randomized clinical study was conducted at Department of Urology of authors' institute over a period of one and half years between January 2014 and June 2015. Informed consent from the patients and approval from ethical committee were obtained. One hundred and ten patients underwent PCNLs during the study period out of which 96 patients who met the inclusion criteria were included in the study. (Figure 1) The reasons for exclusion were multiple percutaneous tracts, significant intraoperative bleeding as judged by the operating surgeons, intraoperative perforation of pelvicaliceal system (PCS) or injury to surrounding structures (pleura, colon, spleen, liver), residual stone where second look was expected, solitary functioning kidney and patient with serum creatinine $>200 \mathrm{micromol} / \mathrm{L}$.

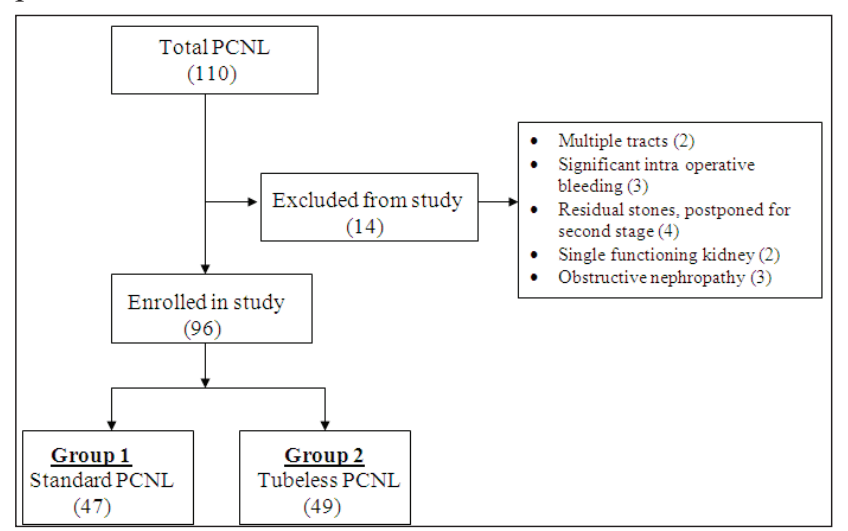

Figure 1: Consort diagram of the study

Patients were evaluated thoroughly with a complete history, physical examination, urinalysis, urine culture and sensitivity test, renal function test, ultrasonography of kidneys, ureter and bladder (KUB) and intravenous urography (IVU) or CT urography. A prophylactic antibiotic (Ceftriaxone 1gm) was administered in all cases before induction of general anesthesia. All PCNLs were performed by the same team of surgeons according to a standard technique.
After the completion of procedure, the double-J (JJ) stent was placed and checked for inclusion and exclusion criteria. All the included patients were randomized into Group 1 (Standard PCNL) and Group 2 (Tubeless PCNL) according to the computer generated random tables which were in sealed envelopes serially and the ratio of allocation in each group was 1:1. In those patients who were allocated to group 1, the procedures were concluded with placement of nephrostomy tube (20-24 Fr) where as in patients in group 2, nephrostomy tubes were omitted and wounds were closed by taking deep sutures using silk 2-0.

Postoperative management was carried out according to department's protocol. Injection piroxicam $40 \mathrm{mg}$ intramuscular was prescribed once daily and pethidine $1 \mathrm{mg}$ per kilogram was kept as per requirement for pain management. Temperature, respiratory rate, pulse rate and blood pressure were measured 4 times a day and when required. The leakage from puncture site, amount, duration and number of dressing changed were noted. Postoperative pain and discomfort were assessed in the next morning using the numerical pain scale (0-10 scale). A total count was done on POD1, POD2 and whenever necessary by automated blood counter. In group 1, nephrostomy tube was removed after 36-48 hrs unless there was need of nephrostomy tube to continue. Routine X-ray KUB on second postoperative day was done to check for residual stones. The variables that were recorded are postoperative fever, systemic inflammatory respond syndrome (SIRS), postoperative pain (assessed by numerical scale: 0-10), analgesic requirement (mg), presence of urinary leak, duration of urinary leak (hours) and length of hospital stay (days).

The data from filled proforma were entered into Statistical Package for Social Sciences (SPSS) 14.0 version (Chicago, Illinois) and analyzed. The continuous variables were expressed as mean $\pm \mathrm{SD}$ and categorical variables as frequency and percentage. Independent t-test and Wilcoxon rank sum test were used to compare the mean of continuous and non-parametric variables respectively. Similarly, Chi square test and Fisher's exact test were used for categorical variables. The confidence interval was set at $95 \%$ and $p$ value $<0.05$ was taken as statistically significant.

\section{Results}

Ninety six patients were eligible for final data analysis. The mean age of the patients was $37.5 \pm 13.5$ years out of which $58(60.4 \%)$ were male. The mean duration of operation was $76.5 \pm 23.3$ minutes.

All the patients' characteristics including age, sex, comorbidities, preoperative investigation parameters, side, size of largest stone, number of stones and mean operation time were comparable in between the groups. (Table 1) 
Table 1: Patient characteristics

\begin{tabular}{|c|c|c|c|}
\hline & $\begin{array}{l}\text { Group } 1 \text { (Standard } \\
\text { PCNL) }(n=47)\end{array}$ & $\begin{array}{l}\text { Group } 2 \text { (Tubeless } \\
\text { PCNL) }(n=49)\end{array}$ & $p$ value \\
\hline Age (Years) & $37.9 \pm 11.3$ & $37.1 \pm 15.5$ & 0.769 \\
\hline Male: Female ratio & $33: 14$ & $25: 24$ & 0.063 \\
\hline Comorbidities & $12 / 47$ & $6 / 49$ & 0.12 \\
\hline Positive urine $\mathrm{C} / \mathrm{S}$ & $4 / 47$ & $3 / 49$ & 0.712 \\
\hline Pre operative hemoglobin (gm\%) & $13.9 \pm 1.5$ & $13.1 \pm 1.8$ & 0.21 \\
\hline Total leukocyte count $\left(/ \mathrm{mm}^{3}\right)$ & $7797 \pm 1564$ & $7305 \pm 2168$ & 0.2 \\
\hline Blood Urea (m mol/L) & $3.7 \pm 0.8$ & $3.6 \pm 1.2$ & 0.744 \\
\hline Serum Creatinine (micro mol/L) & $76.5 \pm 14.5$ & $79 \pm 20.8$ & 0.48 \\
\hline Side= Left: Right & $24: 23$ & $20: 29$ & 0.413 \\
\hline Size of largest stone (mm) & $23.7 \pm 8.7$ & $22.1 \pm 4.8$ & 0.267 \\
\hline Stones=Multiple: Single & $23: 24$ & $19: 30$ & 0.405 \\
\hline Recurrent stone & $9 / 47$ & $5 / 49$ & 0.256 \\
\hline Mean Operative time (min) & $79.9 \pm 22.5$ & $73.3 \pm 23.9$ & 0.168 \\
\hline
\end{tabular}

The incidence of postoperative fever on second POD and SIRS were found to be more in group 1 patients $(29.7 \%$ vs $10.2 \%$; $=0.021$ and $48.9 \%$ vs $16.3 \% ; p=0.001$ ) which were statistically significant. But there was no significant difference noted among the groups on fever on first POD and urosepsis. (Table 2)

Table 2: Post operative fever, SIRS and Urosepsis following PCNL

$\begin{array}{llll} & \begin{array}{l}\text { Group 1 (Standard } \\ \text { PCNL) }(\mathbf{n = 4 7 )}\end{array} & \begin{array}{l}\text { Group 2 (Tubeless } \\ \text { PCNL) (n=49) }\end{array} & p \text { value } \\ \text { Fever- Day 1 (\%) } & 22(46.8 \%) & 17(34.7 \%) & 0.299 \\ \text { Fever- Day 2 (\%) } & 14(29.7 \%) & 5(10.2 \%) & 0.021^{*} \\ \text { SIRS (\%) } & 23(48.9 \%) & 8(16.3 \%) & 0.001^{*} \\ \text { Urosepsis }(\%) & 4(8.5 \%) & 3(6.1 \%) & 0.712\end{array}$

${ }^{*} P$ value $<0.05$ (significant)

Group 2 (Tubeless PCNL) patients had significantly less pain as compared to group 1 (3.6 \pm 1.5 Vs $2.7 \pm 0.8$; $\mathrm{p}=0.001$ ). Standard PCNL (Group 1) patients demanded more injectable opioid analgesic (pethidine) (204.9 $\pm 94.5 \mathrm{mg}$ vs $126.9 \pm 61.8$ $\mathrm{mg}$ ) as compared to group 2 which was statistically significant with $p$ value $<0.001$.

Most of the patients in group 1 (42 patients; 89.4\%) complained of urinary leak from puncture site for a mean duration of $25.9 \pm 11.6$ hours after nephrostomy tube removal. Only one patient $(2 \%)$ had urinary leak from the puncture site in group 2 that is also for 6 hours.

The mean duration of Foley's catheter removal in group 1 and group 2 was $1.8 \pm 0.8$ and $1.7 \pm 0.7$ days respectively and it was not statistically significant $(\mathrm{p}=0.55)$. Nephrostomy tube in group 1 was removed on $2.6 \pm 0.9$ days. The mean length of postoperative hospital stay was more for group 1 as compared to group $2(4.9 \pm 2.1$ vs $3.1 \pm 0.88$ days $)$ which was statistically significant with $p$ value $<0.001$. 
The incidences of postoperative complications and events were comparable in both the groups (Table 3), except for blood transfusion which was found to be more in group $1(14.9 \% \mathrm{Vs} 2 \% ; \mathrm{p}=0.029)$. One patient from each group had persistent hematuria which required CT angiography and selective segmental renal artery embolization. The incidence of perirenal hematoma, urinoma and urinary tract infection were comparable in between the groups.

Table 3: The incidence of post PCNL complications and events

\begin{tabular}{|c|c|c|c|}
\hline & $\begin{array}{l}\text { Group } 1 \text { (Standard } \\
\text { PCNL) }(n=47)\end{array}$ & $\begin{array}{l}\text { Group } 2 \text { (Tubeless } \\
\text { PCNL) }(n=49)\end{array}$ & $p$ value \\
\hline Perinephric hematoma & $1(2.1 \%)$ & $2(4.1 \%)$ & 0.516 \\
\hline Perinephric urinoma & $1(2.1 \%)$ & $0(0 \%)$ & 0.49 \\
\hline Bleeding from puncture site & $2(4.2 \%)$ & $0(0 \%)$ & 0.237 \\
\hline Urinary tract infection & $7(14.9 \%)$ & $3(6.1 \%)$ & 0.194 \\
\hline Blood transfusion & $7(14.9 \%)$ & $1(2 \%)$ & $0.029^{*}$ \\
\hline $\begin{array}{l}\text { Life threatening bleeding requiring } \\
\text { angioembolization }\end{array}$ & $1(2.1 \%)$ & $1(2 \%)$ & 0.742 \\
\hline Re-admission & $3(6.4 \%)$ & $2(4.1 \%)$ & 0.674 \\
\hline
\end{tabular}

${ }^{*} P$ value $<0.05$ (significant)

\section{Discussion}

Traditionally wide bore nephrostomy tube drainage after PCNL has been advocated for several reasons. It provides reliable urinary drainage, hemostatic tamponade to the fresh percutaneous renal tract and continuing access to the renal collecting system should a secondary percutaneous procedure is required. Despite these obvious and important advantages large nephrostomy tubes are thought to contribute to postoperative pain and morbidity. As a result certain investigators have recently proposed tubeless PCNL in an attempt to avoid nephrostomy tube drainage after uncomplicated, straightforward percutaneous procedures. Borges et al. did a systematic review and meta-analysis of 10 randomized controlled trials (RCT) that reported 621 patients and compared tubeless PCNL with standard PCNL. ${ }^{6}$

The incidence of postoperative fever on second POD and SIRS were found to more in group 1; but there was no significant difference noted among the groups on fever in first POD and urosepsis. Even though the advantages of placement of nephrostomy tube, as a drainage conduit to alleviate the sepsis, has been highlighted in the past; recent data shows that tube is a significant contributor for postoperative fever and SIRS. ${ }^{7}$ Fever on first POD could be due to various pyrogenic reactions which were equally distributed in both the groups. There was significant high incidence of fever on second POD and SIRS in that group of patients who had nephrostomy tube. However, the link between the tube and urosepsis could not be established in this study. Various other studies also failed to demonstrate the association of infection / fever with the nephrostomy tube. ${ }^{3,8,9}$ Similarly, with data extracted from six trials, meta-analysis showed no difference between the groups concerning postoperative fever with no heterogeneity between trials. $^{6}$

Tubeless PCNL had significant less pain score as compared to group 1 when assessed by numerical scale on the first postoperative day. Tube placement, especially in the vicinity of a rib, is a significant contributor of pain and discomfort postoperatively. This finding was already accepted by many authors and even in meta-analysis. ${ }^{3,6,9,10}$

Requirement of analgesic has direct relation with the pain score; higher the pain, more would be the demand. The length of hospital stay can also influence it. Various authors had already confirmed it in their series. The meta-analysis of the analgesic requirement in between the groups was not possible to be performed because of a great heterogeneity found in the studies, related to drugs and doses used by the authors. ${ }^{6}$ Whatever the drug or different doses used, one point is common to all that the tubeless PCNL group significantly demanded less analgesic as compared to standard PCNL. 
One of the major reasons to put nephrostomy tube is for urinary drainage, but its use invites post nephrostomy removal urinary leakage from puncture site, which is sometime worrisome to the patients. In this study, almost all patients $(89.4 \%)$ who harbor tube complained of post removal urinary leakage for variable period of time. Only one patient in tubeless group complaint urinary leakage from puncture site, which was also too brief (6 hours) to be mentioned. Nephrostomy tube was removed on an average of $2.6 \pm 0.9$ days and in that time frame a temporary communication between collecting system and skin surface was established. It takes many hours to heal the communication to completely abolish the leakage. This problem was completely absent in tubeless group where the puncture site was closed with deep surgical suture. Some authors also use various sealant materials like- fibrin glue, gelatin matrix / powder to achieve the hemostasis as well as to close the puncture site. ${ }^{11,12}$ In a randomized study of large bore nephrostomy tube, small bore nephrostomy tube and tubeless group, urinary leak was significantly higher in large bore tube category. ${ }^{2}$ His results confirmed that larger the tube, longer the time of urinary leakage from puncture site. In a study of 101 patients, seven had troublesome urinary leakage after tube removal, which stopped spontaneously within 3 to 5 days, while none had that problem in tubeless group. ${ }^{3}$ The incidence of urinary leak has been observed to be lower in the tubeless group than in the conventional group (OR 0.13; CI 95\%: 0.04-0.38, $\mathrm{P}=0.0002)$ with no heterogeneity between trials $\left(\mathrm{I}^{2}=0 \%\right){ }^{6}$

The incidences of postoperative complications and events were comparable in both the groups except for blood transfusion rate, which was more in group $1(14.9 \% \mathrm{Vs}$ $2 \%$ ). A meta-analysis of RCTs didn't show any difference in blood transfusion rate among the standard and tubeless PCNL groups (OR: 0.43; CI 95\%=0.16-1.14; $\mathrm{P}=0.09$ ). ${ }^{6}$ The incidence of life threatening hemorrhage after PCNL necessitating blood transfusion in a larger series is $1.4 \%$ and usually arises from the segmental arteries resulting from the development of arteriovenous fistulas and pseudoaneurysms, which usually occur at an average of 8 days (range 2-18 days) after removal of the nephrostomy tubes. ${ }^{13-15}$ Thus the nephrostomy tube alone cannot blamed for more blood transfusion rate. To draw the final conclusion, we need to have larger multicentric study as the incidence of that event in our series is very small. Infective episodes are more prone with tubes which make patients more vulnerable for secondary bleeding and might need blood transfusion at any time during his treatment. Life-threatening hematuria can be managed by diagnostic angiography and selective angioembolization. ${ }^{13-15}$ Two patients in our study, one each from the two groups required selective angioembolization. Other complications including perinephric hematoma, urinoma and urinary tract infection were comparable in both groups. Readmission after procedure was also comparable in between the groups. (Table 3) This confirmed that tubeless PCNL had similar morbidity as compared to standard PCNL.

Tubeless PCNL patients were discharged from hospital early (3.1 $\pm 0.88 \mathrm{Vs} 4.9 \pm 2.1$ days) as compared to standard PCNL which helped to manage hospital bed more efficiently. Nephrostomy was removed on an average of 2.6 days after PCNL in group 1 and patients remained in hospital for troublesome urinary leakage for variable period of time. Because of these reasons standard PCNL patients stayed for longer post operatively.

The assessor in postoperative period was not blinded in this study (single blinded study), which failed to eliminate the observer bias. Well designed multicentric randomized study, eliminating all possible biases, is required before drawing the final conclusion.

\section{Conclusion}

Tubeless PCNL as compared to standard PCNL is safe in terms of postoperative complications and events. Significant advantages were noted in terms of postoperative fever, SIRS, pain, analgesic requirement, urinary leak from puncture site and length of postoperative hospital stay. Thus, it can be concluded that tubeless PCNL is equally safe as standard PCNL and is found to be less morbid in postoperative period in patients undergoing PCNL.

\section{References}

1. Agrawal MS, Agrawal M. Tubeless percutaneous nephrolithotomy. Indian J Urol 2010;26:1624. https://doi.org/10.4103/0970-1591.60438 PMid:20535280 PMCid:PMC2878433

2. Desai MR, Kukreja RA, Desai MM, Mhaskar SS, Wani KA, Patel SH et al. A prospective randomized comparison of type of nephrostomy drainage following percutaneous nephrostolithotomy: large bore versus small bore versus tubeless. J Urol 2004;172:565-7. https://doi.org/10.1097/01.ju.0000130752.97414.c8 PMid:15247731

3. Agrawal MS, Agrawal M, Gupta A, Bansal S, Yadav A, Goyal J. A randomized comparison of tubeless and standard percutaneous nephrolithotomy. J Endourol 2008;22:439-42. https://doi.org/10.1089/end.2007.0118 PMid: 18257738 
4. Gupta NP, Mishra S, Suryawanshi M, Seth A, Kumar R. Comparision of standard with tubeless percutaneous nephrolithotomy. J Endourol 2008; 22: 1441-6. https://doi.org/10.1089/end.2007.0338 PMid:18690810

5. Bellman GC, Davidoff R, Candela J, Gerspach J, Kurtz S, Stout L. Tubeless percutaneous renal surgery. J Urol 1997; 157: 1578-82. https://doi.org/10.1016/S0022-5347(01)64799-2

6. Borges CF, Fregonesi A, Silva DC, Sasse AD. Systematic review and meta-analysis of nephrostomy placement versus tubeless percutaneous nephrolithotomy. J Endourol 2010;24:1739-46. https://doi.org/10.1089/end.2010.0231 PMid:20958141

7. Luitel BR. Significance of Stone Culture in Percutaneous Nephrolithotomy (PCNL).Thesis (Unpublished) Oct 2009.

8. Feng MI, Tamaddon K, Mikhail A, Kaptein JS, Bellman GC. Prospective randomized study of various techniques of percutaneous nephrolithotomy. Urology 2001;58:345-50. https://doi.org/10.1016/S0090-4295(01)01225-0

9. Sofikerim M, Demirci D, Huri E, Ersekerci E, Karacagil M. Tubeless percutaneous nephrolithotomy: Safe even in supracostal access. J Endourol 2007;21:967-72. https://doi.org/10.1089/end.2006.0216 PMid:17941770

10. Choi M, Brusky J, Weaver J, Amantia M, Bellman GC. Randomized trial comparing modified tubeless percutaneous nephrolithotomy with tailed stent with percutaneous nephrostomy with small-bore tube. J Endourol 2006;20:766-70. https://doi.org/10.1089/end.2006.20.766 PMid:17094752

11. Shah HN, Kausik V, Hegde S, Shah JN, Bansal MB. Initial experience with hemostatic fibrin glue as adjuvant during tubeless percutaneous nephrolithotomy. J Endourol 2006;20:194-8. https://doi.org/10.1089/end.2006.20.194 PMid:16548728

12. Lee DI, Uribe C, Eichel L, et al. Sealing percutaneous nephrolithotomy tracts with gelatin matrix hemostatic sealant: Initial clinical use. J Urol 2004;171:575-8. https://doi.org/10.1097/01.ju.0000103501.98597.b7

\section{PMid:14713762}

13. Srivastava A, Singh KJ, Suri A, et al. Vascular complications after percutaneous nephrolithotomy: Are there any predictive factors? Urology $2005 ; 66: 38-40$. https://doi.org/10.1016/j.urology.2005.02.010 PMid:15992882

14. Kessaris DN, Bellman GC, Pardalidis NP, Smith AG. Management of hemorrhage after percutaneous renal surgery. J Urol 1995;153:604-8. https://doi.org/10.1016/S0022-5347(01)67659-6

15. Martin X, Murat FJ, Feitosa LC, et al. Severe bleeding after nephrolithotomy: Results of hyperselective embolization. Eur Urol 2000;37:136-9. https://doi.org/10.1159/000020129 PMid:10705189 